\title{
Design of a Kalman Filter for a Cutting Force Measurement System
}

\author{
Fu Shao \\ Department of Mechanical and Industrial Engineering, \\ University of Toronto \\ 5 King's College Road \\ Toronto, Ontario, Canada M5S 3G8
}

\begin{abstract}
This paper presents a Kalman filter designed for a cutting force measurement system. The cutting force measurement system is used to measure cutting forces for meso-milling applications. Due to the small magnitude of the cutting forces generated from the meso-milling process, the measurement results are easily distorted by the noise that exists in the measurement system. The object of the Kalman filter design is to reduce the noise influence and obtain accurate cutting force measurement information for the meso-milling applications. The Kalman filter is designed based on an oscillation process model due to the oscillation characteristic of the cutting forces experienced in the meso-milling process.
\end{abstract}

\section{Keywords}

Kalman filter, cutting force measurement, signal filtering, noise reduction

\section{INTRODUCTION}

A novel three-component piezoelectric force sensor has been designed to provide accurate measurement of cutting forces for meso-milling applications [1]. The designed force sensor will be integrated into the overall measurement system, figure 1 , for processing the sensor output signals. The sensor output charge signals are amplified through signal amplification and transformed into digital signals through A/D conversion. The digital signals are then filtered to reduce noise influence and converted into measurement results, namely the estimated cutting force values $\widehat{F}_{X, Y, Z}$.

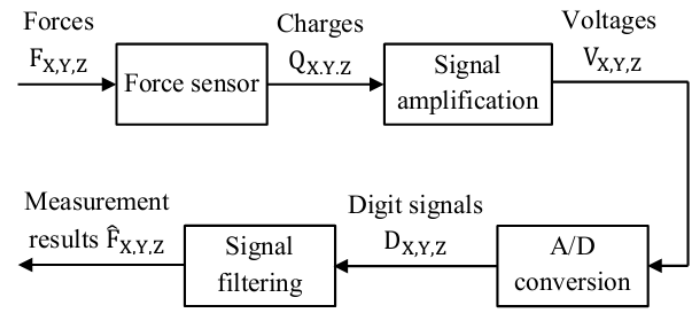

Figure 1: The architecture of the cutting force measurement system.

For the cutting force measurement system, the measurement results not only contain the information of the desired force signals detected by the force sensor but also contain noise. The noise can be acoustic noise emanating from vibration source such as a rotating machine [2], herein the high-speed spindle. The noise can also come from electronic devices [2], such as the signal amplification and A/D conversion circuits. Due to the small parameters used in the meso-milling process, the expected cutting forces are within $1 \mathrm{~N}$ [3] and can go down to $10 \mathrm{mN}$ scale [4]. The measurement results of the subNewton cutting forces can be distorted by noise easily [5] and therefore, signal filtering is necessary and important to the cutting force measurement system. Considering that the cutting forces in meso-milling process are dynamic signals which oscillate at tooth passing frequency [3] and Kalman filter is a pertinent and powerful filtering technique for dynamic signals [2, 6], a Kalman filter is particular designed in this paper for the cutting force measurement system. The paper is organized as: Section 2 presents the designed Kalman filter for the cutting force measurement system; Section 3 analyzes the simulation results of the Kalman filter algorithm; and Section 4 summarizes the conclusion.

\section{KALMAN FILTER FOR THE CUTTING FORCE MEASUREMENT SYSTEM}

Due to the oscillation characteristic of the cutting forces, the Kalman filter is designed based on an oscillation process model [7]:

$$
\ddot{y}(t)+\omega^{2} y(t)=u(t),
$$

where $y(t)$ represents the cutting force signal, $\omega$ represents the oscillation frequency of the cutting forces, and $u(t)$ represents the noise that exists in the cutting force measurement which can be assumed as white noise [5]. Since the process model is a second-order differential equation, the state vector for the cutting force measurement is a two-tuple [7]. Accordingly, Eq. (1) is rewritten in state space format as:

$$
\begin{gathered}
\dot{\mathbf{x}}(\mathrm{t})=\mathbf{F} \mathbf{x}(\mathrm{t})+\mathbf{G u}(\mathrm{t}), \\
\mathrm{y}(\mathrm{t})=\mathbf{B x}(\mathrm{t}),
\end{gathered}
$$

where $\mathbf{x}(t)=\left[\begin{array}{l}x_{1}(t) \\ x_{2}(t)\end{array}\right]$ represents the state vector for the cutting force measurement with $\mathrm{x}_{1}(\mathrm{t})$ represents the value of the oscillating force signal, i.e., $\mathrm{x}_{1}(\mathrm{t})=\mathrm{y}(\mathrm{t})$, and $\mathrm{x}_{2}(\mathrm{t})$ represents the changing velocity of $\mathrm{x}_{1}(\mathrm{t})$, i.e., $\mathrm{x}_{2}(\mathrm{t})=\dot{\mathrm{x}}_{1}(\mathrm{t})$, as illustrated in figure 2. The associated transition matrix $\mathbf{F}$ and transition vectors $\mathbf{G}$ and $\mathbf{B}$ are, respectively:

$$
\begin{gathered}
\mathbf{F}=\left[\begin{array}{cc}
0 & 1 \\
-\omega^{2} & 0
\end{array}\right], \\
\mathbf{G}=\left[\begin{array}{l}
0 \\
1
\end{array}\right], \\
\mathbf{B}=\left[\begin{array}{ll}
1 & 0
\end{array}\right] .
\end{gathered}
$$$$
\mathrm{U}(\mathrm{S}) \longrightarrow \frac{\mathrm{S}}{\mathrm{S}^{2}+\omega^{2}} \stackrel{\mathrm{X}_{2}(\mathrm{~S})}{\longrightarrow} \stackrel{\frac{1}{\mathrm{~S}}}{\stackrel{\mathrm{X}_{1}(\mathrm{~S})}{\longrightarrow} \mathrm{Y}(\mathrm{S})}
$$

Figure 2: The block diagram for the state model of the cutting force measurement. 


$$
\mathbf{Q}=\left[\begin{array}{ll}
\int_{0}^{\Delta \mathrm{t}} \int_{0}^{\Delta \mathrm{t}} \mathrm{g}_{1}(\xi) g_{1}(\eta) E[u(\xi) u(\eta)] d \xi d \eta & \int_{0}^{\Delta \mathrm{t}} \int_{0}^{\Delta \mathrm{t}} \mathrm{g}_{1}(\xi) g_{2}(\eta) E[u(\xi) u(\eta)] d \xi d \eta \\
\int_{0}^{\Delta \mathrm{t}} \int_{0}^{\Delta \mathrm{t}} g_{1}(\xi) g_{2}(\eta) E[u(\xi) u(\eta)] d \xi d \eta & \int_{0}^{\Delta \mathrm{t}} \int_{0}^{\Delta \mathrm{t}} g_{2}(\xi) g_{2}(\eta) E[u(\xi) u(\eta)] d \xi d \eta
\end{array}\right]
$$

Assuming that the cutting force measurement starts from a stationary state before the cutting process, the initial value of the state vector is defined as:

$$
\mathbf{x}(0)=\left[\begin{array}{l}
\mathrm{x}_{1}(0) \\
\mathrm{x}_{2}(0)
\end{array}\right]=\left[\begin{array}{l}
0 \\
0
\end{array}\right]
$$

Relating to the sampling process in the cutting force measurement, the discrete process and observation models are defined for the sampling process as:

$$
\begin{gathered}
\mathbf{x}(\mathrm{k})=\boldsymbol{\phi} \mathbf{x}(\mathrm{k}-1)+\mathbf{w}_{\mathrm{k}} \\
\mathrm{z}(\mathrm{k})=\mathbf{H x}(\mathrm{k})+\mathrm{v}_{\mathrm{k}},
\end{gathered}
$$

where $\mathbf{x}(\mathrm{k})=\left[\begin{array}{l}\mathrm{x}_{1}(\mathrm{k}) \\ \mathrm{x}_{2}(\mathrm{k})\end{array}\right]$ and $\mathbf{x}(\mathrm{k}-1)=\left[\begin{array}{l}\mathrm{x}_{1}(\mathrm{k}-1) \\ \mathrm{x}_{2}(\mathrm{k}-1)\end{array}\right]$ are the process state vectors at discrete sampling points $t_{k}$ and $t_{k-1}$, respectively; $\boldsymbol{\phi}$ represents the transition matrix relating $\left[\begin{array}{l}\mathrm{x}_{1}(\mathrm{k}-1) \\ \mathrm{x}_{2}(\mathrm{k}-1)\end{array}\right]$ to $\left[\begin{array}{l}\mathrm{x}_{1}(\mathrm{k}) \\ \mathrm{x}_{2}(\mathrm{k})\end{array}\right] ; \mathbf{w}_{\mathrm{k}}$ represents the noise sequence that accompanies the input force signal in the sampling process; $\mathbf{H}$ represents the measurement vector; $\mathrm{z}(\mathrm{k})$ represent the measurement result at sampling point $t_{k}$, and $v_{k}$ represents the measurement error. $\boldsymbol{\phi}$ and $\mathbf{H}$ are defined as:

$$
\begin{gathered}
\boldsymbol{\phi}=\left\{\mathcal{L}^{-1}\left[(\mathrm{~s} \mathbf{I}-\mathbf{F})^{-1}\right]\right\}_{\mathrm{t}=\Delta \mathrm{t}} \\
=\left[\begin{array}{cc}
\cos (\omega \Delta \mathrm{t}) & \frac{1}{\omega} \sin (\omega \Delta \mathrm{t}) \\
-\omega \sin (\omega \Delta \mathrm{t}) & \cos (\omega \Delta \mathrm{t})
\end{array}\right], \\
\mathbf{H}=\mathbf{B}=\left[\begin{array}{ll}
1 & 0
\end{array}\right] .
\end{gathered}
$$

where $\Delta \mathrm{t}$ is the time interval used in the sampling process. The covariance of the noise signals $\mathbf{w}_{\mathrm{k}}$ and $\mathrm{v}_{\mathrm{k}}$ is given as [7]:

$$
\begin{gathered}
\mathrm{E}\left[\mathbf{W}_{\mathrm{k}} \mathbf{W}_{\mathrm{i}}^{\mathrm{T}}\right]=\left\{\begin{array}{ll}
\mathbf{Q}, & \mathrm{i}=\mathrm{k} \\
0, & \mathrm{i} \neq \mathrm{k}
\end{array},\right. \\
\mathrm{E}\left[\mathrm{v}_{\mathrm{k}} \mathrm{v}_{\mathrm{i}}^{\mathrm{T}}\right]=\left\{\begin{array}{ll}
\mathrm{R}, & \mathrm{i}=\mathrm{k} \\
0, & \mathrm{i} \neq \mathrm{k}
\end{array},\right. \\
\mathrm{E}\left[\mathbf{W}_{\mathrm{k}} \mathrm{v}_{\mathrm{i}}^{\mathrm{T}}\right]=0,
\end{gathered}
$$

where $\mathbf{Q}$ is a covariance matrix and $\mathrm{R}$ is defined as the variance of the measurement error. Following the transfer function approach [7], the matrix $\mathbf{Q}$ is defined as:

where functions $g_{1}(t)$ and $g_{2}(t)$ are defined from figure 2 as:

$$
\begin{aligned}
& g_{1}(t)=\mathcal{L}^{-1}\left[\frac{X_{1}(S)}{U(S)}\right]=\mathcal{L}^{-1}\left[\frac{1}{S^{2}+\omega^{2}}\right]=\frac{1}{\omega} \sin \omega t \\
& g_{2}(t)=\mathcal{L}^{-1}\left[\frac{X_{2}(S)}{U(S)}\right]=\mathcal{L}^{-1}\left[\frac{\mathrm{S}}{S^{2}+\omega^{2}}\right]=\cos \omega t
\end{aligned}
$$

and the autocorrelation of the white noise $u(t)$ results in a delta function:

$$
\mathrm{E}[\mathrm{u}(\xi) \mathrm{u}(\eta)]=\sigma^{2} \delta(\xi-\eta)
$$

where $\sigma$ represents the standard deviation of the white noise. Substitute Eq. (16) to Eq. (18) into Eq. (15) and utilize the property of delta function in the integration, the matrix $\mathbf{Q}$ is determined for sampling process as:

$\mathbf{Q}=\frac{\sigma^{2}}{4 \omega^{3}}\left[\begin{array}{cc}2 \omega \Delta t-\sin (2 \omega \Delta t) & 2 \omega \sin ^{2}(\omega \Delta t) \\ 2 \omega \sin ^{2}(\omega \Delta t) & 2 \omega^{3} \Delta t+\omega^{2} \sin (2 \omega \Delta t)\end{array}\right]$.

The function of the Kalman filter is to estimate current state vector $\hat{\mathbf{x}}(\mathrm{k})$ (corresponding to current input force value) from current measurement result $\mathrm{z}(\mathrm{k})$ and previous estimate $\hat{\mathbf{x}}(\mathrm{k}-1)$. The implementation of the Kalman filter comprises of initialization and recursion processes, figure 3 . The initialization defines the estimated initial state $(k=0)$. The recursion process (for $\mathrm{k} \geq 1$ ) comprises of a series of computation steps which form a recursion loop for performing the filtering function. Relating to Eq. (7), the estimated initial state is defined as:

$$
\hat{\mathbf{x}}(0)=\left[\begin{array}{l}
\hat{\mathrm{x}}_{1}(0) \\
\hat{\mathrm{x}}_{2}(0)
\end{array}\right]=\left[\begin{array}{l}
0 \\
0
\end{array}\right],
$$

and the error covariance related to this estimate is:

$$
\mathbf{P}(0)=\mathrm{E}\left[(\mathbf{x}(0)-\hat{\mathbf{x}}(0))(\mathbf{x}(0)-\hat{\mathbf{x}}(0))^{\mathrm{T}}\right]=\left[\begin{array}{ll}
0 & 0 \\
0 & 0
\end{array}\right] .
$$

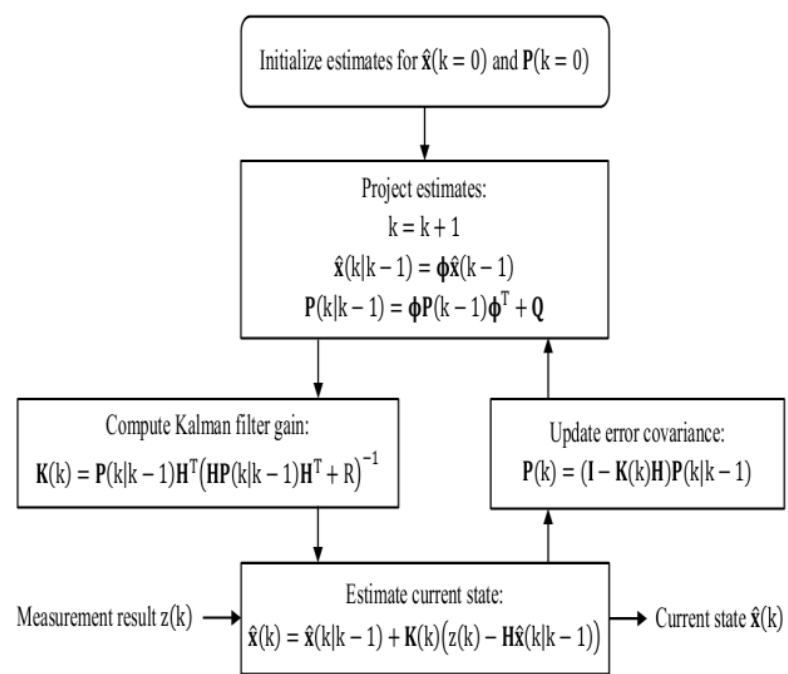

Figure 3: Initialization and recursion processes of the Kalman filter 


\section{SIMULATIONS AND ANALYSIS}

Simulations based on figure 3 were performed in MATLAB ${ }^{\circledR}$ software environment with respect to the proposed cutting force measurement. The variance $\mathrm{R}$ was set as 0.25 in the simulations, namely the standard deviation of the measurement error was assumed to be one-half in evaluating the performance of the designed Kalman filter. The standard deviation $\sigma$ was set as $0.02,0.03$, and 0.04 , respectively, to correspond with different levels of the noise sequence that are supposed to accompany the input force signals in proposed cutting force measurement, as shown in figures 4 to 6 for the three relating noise levels. Simulation result for an actual input force value of $10 \mathrm{mN}$ with respect to the minimum amplitude in the measurement range [1] is presented in figure 4 for a noise level of $20 \mathrm{mN}$ which has been experienced in the measurement of cutting forces in meso-milling process [3]. It can be seen from the figure that the simulated measurement result without the designed Kalman filter shows random characteristic due to the noise influence while the simulated measurement result with the designed Kalman filter follows the simulated actual input force signal accurately. Namely, the noise influence has been eliminated by the designed Kalman filter. Simulations with higher noise levels also demonstrate the same conclusion, as shown in figure 5 and figure 6 for examples.

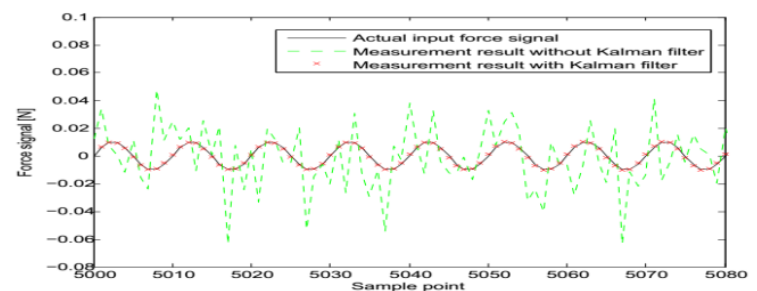

Figure 4: Kalman filter used for the measurement of cutting force with amplitude of $10 \mathrm{mN}$ and an input noise level of $20 \mathrm{mN}$

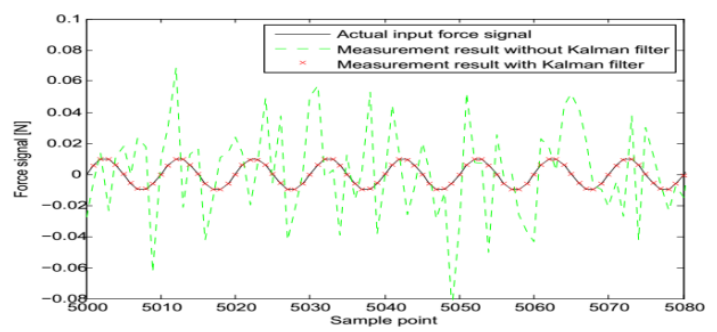

Figure 5: Kalman filter used for the measurement of cutting force with amplitude of $10 \mathrm{mN}$ and an input noise level of $30 \mathrm{mN}$

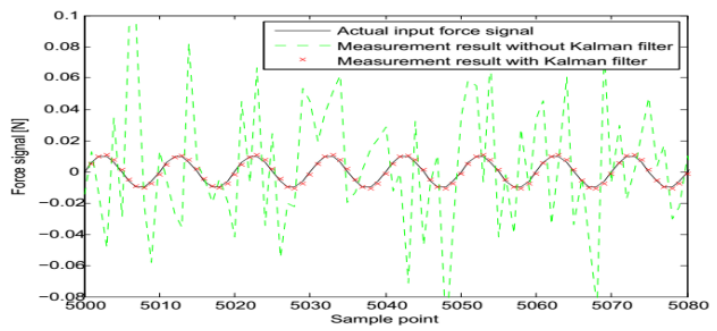

Figure 6: Kalman filter used for the measurement of cutting force with amplitude of $10 \mathrm{mN}$ and an input noise level of $40 \mathrm{mN}$

\section{CONCLUSION}

The Kalman filter based on an oscillation process model has been designed in this paper for a cutting force measurement system for use in meso-milling process. Due to the small magnitude of the cutting forces generated from the mesomilling process, the cutting force measurement results are easily distorted by the noise that exists in the measurement system. The objective of the Kalman filter design is to reduce the noise influence on the measurement results and provide accurate cutting force measurement information for mesomilling applications. Simulations with respect to the designed Kalman filter were performed for different noise levels and the results show that the measurement results with the designed Kalman filter follow the actual input force signals accurately. The future work will focus on the integration of the designed Kalman filter into all the three channels of the cutting force measurement system for use in the measurement of the three-components, $\mathrm{F}_{\mathrm{X}, \mathrm{Y}, \mathrm{Z}}$, of the cutting forces for meso-milling applications.

\section{ACKNOWLEDGMENT}

This research is funded by the Natural Science and Engineering Research Council of Canada through the Canadian Network for Research and Innovation in Machining Technology (NSERC CANRIMT).

\section{REFERENCES}

[1] F.Shao, Design of a Three-Component Force Sensor forMeso-Milling Applications, University of Toronto: PhD Thesis, 2015.

[2] S. V. Vaseghi, Advanced Digital Signal Processing and Noise Reduction, Chichester; New York: John Wiley, 2008.

[3] M. P. Vogler, X. Liu, S. G. Kapoor and R. E. Devor, "Development of meso-scale machine tool (mMT) systems," Transactions of NAMRI/SME, pp. 653-661, 2002

[4] G. Bissacco, T. Gietzelt and H. N. Hansen, "Force analysis in micro milling $\mathrm{Al} 6082 \mathrm{~T} 6$ in various engagement conditions," in 4th International Conference on Multi Material Micro Manufacture (4M), Cardiff, United Kingdom, 2008.

[5] J. Chae and S. S. Park, "High frequency bandwidth measurements of micro cutting forces," International Journal of Machine Tools \& Manufacture, vol. 47, no. 9, p. 1433-1441, 2007.

[6] G. Evensen, Data Assimilation: The Ensemble Kalman Filter, Berlin; New York: Springer, 2007.

[7] R. G. Brown and P. Y. Hwang, Introduction to Random Signals and Applied Kalman Filtering: with MATLAB Exercises and Solutions, New York: Wiley, 1997. 